\title{
THE TREATMENT OF FRACTURES.
}

A Lecture during the Winter Course of Training, 1935-36, Malta.

By Brevet Lieutenant-Colonel D. C. MONRo. Royal Army Medical Corps.

I HAVE chosen the subject of fractures as my contribution to our course of lectures this winter, not in an effort to live up to my title of "local expert"-few, indeed, can call themselves expert in such a vast subjectbut rather because, in the first place, I thought a professional subject was expected of me, and secondly, that by indicating in a general way the tendencies in more recent methods of treatment, and the beliefs on which these methods are based, my remarks might be of some assistance to those whose time is taken up more with administration, or the study of medical subjects in general.

It is quite unnecessary for me to remind you of the deformity and serious crippling, particularly in the case of limbs, that a badly treated fracture leads to. In the Service, such a state of affairs would invariably lead to invaliding, and all its consequences-unemployment, cost to the State, etc. A badly treated fracture is the very worst form of "walking or rather hobbling advertisement," and to anyone with only half a conscience responsible for such a result should be a subject for selfincrimination.

Only within comparatively recent years has the profession become sufficiently alive to the fact that sometbing had been lacking in the general conduct of fracture cases in medical institutions: witness the comparatively recent Commission on the Treatment of Fractures, the increase in numbers of orthopædic specialists and consultants, the establishment of special fracture clinics, and the spate of writing on the subject in medical journals.

The more senior of us will remember what scant and sketchy instructions-compared with modern teaching-were given at medical schools, when we were students. I believe that it would not be far wrong to state that the more rational systematic instruction on the subject is the product of post Great War years.

Twenty years ago a fracture case was regarded as a slightly unwelcome and rather annoying occupant of a bed which might well accommodate an interesting "abdomen." The usual examination was of course carried out, and as much reduction as possible effected, all efforts being directed towards getting the case out and home in an ambulatory splint as soon as possible. The after-treatment of the case was, to say the least of it, haphazard, and too often left to a busy general practitioner, who "did his best." The more fortunate cases were followed up to some extent, the after-treatment 
consisting mainly of periodic adjustment of splints, and after a long and tedious period of inactivity on the part of the injured limb, the case would be handed over to the "tender mercies" of some burly masseur, who based his treatment too often on the assumption that the patient was not trying, or chicken-hearted, and considered the forcible moving of stiff joints and the breaking down of adhesions his main object and raison d'être.

Fifty years ago Lucas-Championnière establisbed a reputation in Paris, largely because he discarded irksome splints early and employed massage. He based his methods on the theory that a certain amount of movement at the site of fracture stimulated union: he even employed local pounding and hammering directed at the fracture site from the extremity of a long bone. Speaking confidentially on the secret of his success as a world-renowned masseur be said the reason is : "I never hurt a patient" (Mennell). As judged by the standards of those days, his results appear to have been good, and most certainly his patients thoúght much of bim. Many years later, Arbuthnot Lane, advocating that accurate reduction was the crux of the question of treatment, and malsing use of a rigid technique of asepsis, such as Lister could scarcely have visualized, reduced most fractures by open operation under direct vision, retaining the fragments in position by internal splints. His results were apparently excellent, at all events in cases in which the internal splint, usually a metal plate, was well tolerated. All surgeons recognized the paramount importance of overcoming the pull of tonic and shortening muscles by some form of fixation or constant extension, and much ingenuity was exhibited in attempts to attain this end. But, while all methods resulted in many excellent recoveries, those baffling cases in which non-union occurred continued to be reported. These failures gave the "bone carpenters" their chance, and technique in bone grafting, wiring, pegging or clamping of fragments, reached high standards.

Then came the Great War. The vast numbers of cases, the majority compound, comminuted and nearly always severely infected, provided a large field for further study. Our own Corps was well to the fore here, and the names of Maurice Sinclair, Pearson and several others will go down in the history of surgical endeavour for the excellence of their methods of treatment. I feel that I can say with confidence that, at the present time, the results of treatment of fractures in the Army can compare more than favourably with the results of such injuries treated in civil life. Of course, most of our. cases are fit young men, and all of us appreciate what necessary adjuncts bodily health and vigour are if a fracture is to unite. But-there is still room for improvement.

Where cause and immediate effect are so obvious, empiricism should find no place in treatment : our methods should be based on knowledge of establishéd facts, and backed by experience. Great strides have been made during the last few years, and while the advances can be put down largely to the increase in specialism and in fracture clinics and depart- 
ments, a better understanding of the physiology of ossification, and pathology of bone growth, has contributed most. Without some idea of this rather complex but most interesting subject, we must be working largely in the dark. I shall endeavour to attempt to present to you some of the facts and theories relating to the subject as succinctly as possible, merely accentuating main points. Let me state here that I shall quote almost entirely from "Surgical Pathology" (Illingworth and Dick) and Hey Groves' translation of Bohler's book on " Treatment of Fractures."

Most bones are developed from a previous model in cartilage, the process of normal ossification taking place in a constant order for each bone, at constant age-periods, from what are known as centres of ossification. If we take a typical long bone as an example, it consists of a shaft (or diaphysis) and two extremities (or epiphyses) and occasionally accessory prominences (or apophyses). There is a centre for each such subdivision and during the process of growth the epiphysis is separated from the diaphysis by a zone of hyaline cartilage, the epiphyseal cartilage or plate. Except at its articular ends the whole bone is clothed by a fibrous vascular membrane-the periosteum, which is attached most firmly round the growing bone at the region of the epiphyseal cartilage. From the point of view of the pathologist in particular, one of the most important areas of a growing bone is the metaphysis, or area of the shaft, immediately adjoining the epiphysis. It is here, during the period of growth, that the circulation is richest and where the vascular spaces are numerous, thin-walled and dilated, so that they are particularly prone to injury and congestive stasis, and form a ready trap for infective organisms circulating in the blood. This is the area in which acute osteomyelitis almost invariably commences. Growth in any tissue is dependent on an adequate blood supply. Blood in the case of a bone reaches the zone of growth, not only by the nutrient vessels but also from the periosteum and from an intimate periarticular anastomosis.

It should be remembered that cartilage does not ossify, but merely provides a cellular matrix for the formation of the necessary embryonic type of connective tissue (mesenchymé) in which ossification takes place by replacement.

The roll of the skeleton is not merely supporting. It acts as a storehouse for the elements calcium and phosphorus-both essential to life-and keeps these substances at a constant level in the blood-stream, a state of chemical equilibrium in which the secretions of the pituitary and parathyroid glands and vitamin $\mathrm{D}$ play a directing part. It is most important to keep in mind that bone is not an inert immutable framework but a living tissue, liable to modelling and remodelling processes to meet the stresses imposed by muscle pull and gravity, and capable of repairing the damage resulting from disease or trauma.

The roll of the periosteum has long been a subject for discussion and argument, but most observers now agree with Macewen of Glasgow, that 
this membrane should be regarded as a "limiting barrier to ossification.". Foremost among the issues in this "battle of the periosteum" is the function of the osteoblast. For a long time it was believed that the tissue bone was the result of the specific vital activities of the osteoblast, and that in the absence of osteoblasts no osseous tissue could be formed. A majority, however, now consider that the function attributed to the osteoblast alone can be assumed by any primitive mesenchymal cell, under certain conditions of environment. This, the theory of Lariche and Policaard, denies the existence of a specific bone-forming cell, while maintaining that bone, cartilage, and even fat and tendons are the derivatives of a process of metamorphosis (metaplasia) in primitive mesenchyme, whereby the cells are "adapted" to suit the local needs and functional requirements, basing their belief on the study of a large amount of anatomical and pathological material.

The champions of the theory maintain that bone formation must always occur in such primitive tissue which they designate an "ossifiable medium," provided that there is an adequate and readily available supply of calcium salts and that certain otber factors, depending on conditions of blood and lymph circulation, are favourable (viz. the presence of the ferment phosphatase and the correct $\mathrm{pH}$ value in the serum). To sum up, all changes preparatory to the formation of bone depend on local adjustments of the circulation, its calcium content, local $\mathrm{pH}$ value, etc., and are quite independent of the presence of osteoblasts. For example, it is known that bone may form in scar tissue in the abdominal wall (some distance from the nearest bone), in glands (parotid), in muscle ("rider's bone"), and even in the walls of aneurysmal sacs.

Bone resorption (atrophy or osteoporosis) takes place as the result of the physico-chemical process called "balisteriasis" in which calcium salts are withdrawn by the body circulating fluids through the agency of the large foreign body type of giant cells called osteoclasts.

How do these theories fit into our mental picture of what happens during the bealing of a fracture? The injury occurs, the local soft tissues are lacerated and contused, the bone is fissured or comminuted, and ruptured blood-vessels permit extravasation. The area of the fracture becomes "the fracture sac" in which the blood sooner or later clots. In the course of a few days the clot begins to organize, and becomes replaced by granulation tissue, or in other words young connective tissue of primitive type. There is traumatic inflammation with byperæmia; the byperæmia and protective disuse of the part result in bone absorption, greatest in the fractured ends, with liberation of a local excess of calcium salts. As organization proceeds the inflammation subsides and there is the usual slowing up of the circulation in the area (odema), due to the fact that the new local blood system is not yet properly established. Now we have the correct conditions for fresh deposition of calcium salts, and so primary, and later secondary, callus is formed. Still later, under the stresses of the pull 
of muscles, once more brought into use, of gravity, and weight-bearing, the secondary callus is remodelled into something permanent, which in a well-treated fracture may well be indistinguisbable from the segment of the bone as it was before injury.

Under normal conditions callus begins to appear about the tenth day, and is complete in approximately twenty-one days. Consolidation of callus varies in different sites, but in weight-bearing bones it may well take four to six months.

The study of bone pathology enables us to arrive at-inter alia-two conclusions which are of importance to us when considering fractures, and upon which it is possible to formulate two most important principles.

(a) That increased circulation (hyperæmia) practically always leads to bone absorption (osteoporosis, halisteriasis). Examples: Bone in the early stages of acute osteomyelitis; the ends of fragments in a fracture fourteen days after the injury; the zone of bone immediately adjoining a vascular new growth, etc.

(b) That circulatory stasis or congestion or odema, will lead to bone sclerosis, particularly if the stasis follows a period of hypercemia. Examples: the ends of fragments of a fracture in the later healing of a fracture; the density of bone in an involucrum, or adjoining a chronic pyogenic bone abscess.

It is on the study of the mineral (calcium) salt concentration, as evidenced by the density of shadow cast on X-ray films, that we are able to watch and "check up" the progress of healing in fractures.

\section{Non-Union in Fractidres.}

I have already alluded to this type of case as "baffling," but with the more complete knowledge of the subject that we now possess, the element of mystery surrounding such an untoward result can usually be completely eliminated. We learn by the study of our failures.

Briefty the causes of non-union can be tabulated as : (1) General; or (2) local.

(1) General causes.-Under this beading come such conditions as preexisting systemic disease (syphilis, rickets, etc.) and avitaminosis; blood diseases, etc.

(2) Local causes.-Mechanical : Interposition of a formed tissue barrier (viz. strip of muscle, fascia, periosteum, or a sclerosed and semi-detached fragment) ; over-extension; non-apposition of fragments ; actual bone loss.

Non-mechanical : Retained sepsis ; persistent inflammation (hyperæmia) due to retained foreign body (such as a bone plate); excessive movement; injury to the principal local blood supply; pathological causes (unsuspected tumour or bone cyst, etc.).

\section{Treatment.}

With the time at my disposal I can deal with treatment only on general lines. 
Every fracture should be regarded as a surgical emergency. First aid is necessarily limited to relief of pain and shock and to the application of any available means of steadying the fragments until such time as the case can be got to hospital, thoroughly examined, and appropriate treatment initiated. Radiological examination is important and in most cases an absolute necessity.

The essential problem in the treatment of all fractures is the provision of a substitute for the damaged skeletal support ; this substitute must be capable of maintaining the fragments in their reduced position, inumobilized until such time as union shall occur, and functional recovery be well advanced. I propose to attack the problem under the headings: (1) Reduction; (2) retention; and (3) recovery of function.

(1) Reduction.-Naturally our aim is to replace the fragments as accurately as possible. The great bar to accurate reduction is muscle spasm. Every movement in a recent fracture causes pain and the pain . produces reflex spasm in the adjacent and related muscles. This is a protective mechanisn.

Muscle spasm is caused only by pain, and can be prevented if pain be abolished. Efforts at reduction should therefore be made under the influence of anæsthesia, and whenever applicable, local anæsthesia is far the best form to use. It is also remarkable that the very great shock that results from severe fractures can be eliminated within a few minutes by infiltrating the fracture area with a local anæsthetic. We all realize that accurate reduction is most difficult to attain in certain fractures, even after repeated attempts controlled by screening, but only in a very limited number should it be found necessary to resort to open reduction in a simple fracture. Apart from the special problems which arise in a particular fracture, it is useful to keep in mind that in the case of limbs the fragments will align best when the limb is in a position half way between flexion and extension, and that in the region of ball-and-socket joints the longer distal fragment will usually have to be brought into alignment with the shorter proximal fragment under control of a group of muscles, the combined action of which, when lacking the resistance they normally expect, is to displace the fragment mainly in one direction.

(2) Retention.- Whereas reduction may often be easy, retention is always difficult. Even when reflex spasm has been abolished we still have to deal with the normal tone or tension in the muscles; a property which results in the "taking up of any slack" when their normal tension is disturbed by lack of apposition, or insecurity of normal attachment or insertion. This, so called "adaptive shortening," causes contractures, and will cause displacement of fragments or angulation at the site. Retention must therefore be not only constant, but prolonged. It is usual to have to continue immobilization in a fracture of the radius for six weeks, or of the tibia, for ten weeks, when progress is quite normal.

(3) Recovery of Function.-This is the goal to which all our efforts are 
directed. There is little merit in firm union without recovery of full function. Function implies usefulness, and without recovery of usefulness any method of treatment is useless. We know that an inflamed limb is painful, and that it is held naturally in a position of rest; without fixation, a broken limb remains painful, not only at the site of fracture, but at the related joints. Fixation and immobilization of a limb lead to "disuse atrophy" ; there is lymph-vascular congestion, absorption of the substance of the muscles, and extraction of lime salts from the bones. But, if complete fixation can be accompanied by as much active movement in the related joints and muscles as can be undertaken without causing pain, then one can be sure that function will be very soon recovered when the fixation is discontinued:

With reference to this problem, Bohler states: "These conditions can be fulfilled by the proper arrangements of technical apparatus . . . it does not matter whether it is done by wooden or metal splints, plaster casts, traction apparatus or expensive moulded splints. It is only of. importance that fixation should be effective and continuous and not removed too soon. The best, cheapest and most comfortable fixation-material for most fractures is plaster of Paris. A plaster exo-skeleton can be made to fulfil the function of the damaged endo-skeleton. Natural use of a muscle renders superfluous that poor substitute, massage."

Bohler uses unpadded plaster splints from the outset, and these may bave to be changed as soon as they become loose after the subsidence of swelling. Under this splint the muscles are encouraged to move, the circulation being thus kept up to almost full pressure.

Padded splints, no matter how carefully applied, permit movement of the fragments.

The unpadded splint should always be applied without previous shaving or greasing of the skin.

In the case of the lower limb, an inset iron, distributing the weight to the proximal fragment, makes the splint ambulant.

Late After-treatment.-Within the first week or ten days, a plaster splint may require bi-valving because of traumatic œdema, but this can be prevented or reduced to a negligible quantity by elevation of the limb. Should the plaster become loose, it should be changed ; one half is removed at a time and a fresh "slab" applied, the otber side being changed as soon as the new slab has set.

Splints should never be renoved too soon with the object of permitting local massage and passive movements. Pain will invariably be felt if this is done. Pain should be regarded as evidence of torture, and not as a natural feature in the process of recovery. Passive movements, particularly if they cause pain, will increase stiffness, not reduce it. With active movements the reverse is the case, and such volitional movements may be assisted, provided such assistance causes no pain.

Edema persisting after removal of splints may be quickly reduced by 
the application of pressure bandages, such as zinc-gelatin or elastoplast, radiant heat, and massage.

Delayed or faulty union occurs even in simple fractures, as most of us know to our cost. According to different observers, the frequency of this occurrence is $\frac{1}{2}$ to 6 per cent. Strangely enough it happens most often in strong healthy males; the middle of the radius and lower third of the tibia are common sites. The causes are the same as those which lead to non-union.

In pseudo-arthrosis the medullary cavity of each fragment becomes obliterated by sclerosed bone, the ends pointed and separated by a mass of scar tissue with defective blood-supply.

In dealing with this class of case, Bobler states that he has treated 12,000 of them and has never used any of the much vaunted internal medicaments. He believes that good normal food with adequate vitamin content is necessary, just as it is necessary to treat systemic disease if present. He operates on cases of pseudo-arthrosis, exposing the fracture ends, removes sclerosed bone and scar tissue, drills each fragment diagonally in numerous places, and apposes them again, with or without a Woolf's graft. These measures, combined with careful immobilization, amount simply to the provision of fresh channels for a new blood supply to become established at the site.

\section{Compound Fractures.}

Unfortunately, time will permit only brief reference to this class of case. I can merely mention the methods Bohler applies at his clinic.

In pre-Lister days, Volkmann estimated the mortality from compound fractures at 40 per cent, and it would not be far wrong to assume that apart from an odd case of shock, hæmorrhage, or embolism, the majority died from septic infection or secondary hæmorrhage. It is obvious that compound fractures must be regarded as emergencies. In the more severe cases the first indications will be to stop hæmorrhage and treat shock. The most certain way to accomplish relief of shock is to infiltrate the area with a local anæsthetic. The improvement in a patient's condition soon after this has been done is often miraculous.

Do not shave, scrub, or wash the area of the wound, simply remove gross dirt using a swab moistened with ether or spirit, and paint with tr. iodi. Carry out excision of badly damaged skin edges and muscle, preferably using a tourniquet. Bleeding points should be stopped by pressure or diathermy. Spare tendons. Approximate the ends of divided nerves by suture. When severed muscles retract, they leave undermined skin, and the wound may have to be enlarged; incisions for this purpose should be made in a longitudinal direction. Soiled bone edges should be removed with a sharp chisel or nibbling forceps. Loose fragments of bone should be removed only when severed from all attachments. On completion of this "debridement," treat the wound with iodine and then suture the 
skin only, using tension relief incisions if necessary. Drain obvious dead spaces for forty-eight hours.

The fracture is now dealt with as if it were a closed one. Displacement is treated by screw traction, and plaster slabs, with "windows" applied, or transfixion pins are incorporated in the plaster.

No dressing is applied. The blood and serum escaping from the wound make an ideal dressing. Anti-tetanus and anti-gas gangrene serum may be given, according to the degree of soiling. To obviate undue swelling, the limb is elevated. Later, should it be obvious that suppuration is occurring, only enough sutures are removed to allow the escape of discharge under tension, and the drainage of deep pockets. Otherwise sutures are left: in situ for three weeks. Should subsequent X-ray examination reveal sequestra, these should not be removed before three to six months.

The hospital in Vienna, where Bohler is chief of a skilled and experienced staff, treats practically nothing but cases of accidental injury. It is financed by the local insurance companies. The directors of these corporations were shrewd enough to realize that it paid handsomely to finance such an institution.

In my humble opinion the principles on which Bohler works are preeminently sound. 'I must refer you to Hey Groves' excellent translation of Bohler's book for the methods in which he applies the principles in treatment. I am also convinced that his methods should be generally adopted in the Medical Services of the Army. The fact that much of the apparatus used by the Vienna clinic, or modified by those working on the same lines, has recently been authorized as part of our surgical armament is clear indication that this is also the opinion of our Consultative Committee at the War Office. What a change from twenty years ago! May I quote from my Annual Surgical Report from a large station in India in 1921:-

"On a recent emergency visit to operate in a large out-station, the O.C. Hospital informed me that he had no thigh splints, Thomas pattern or otherwise. This seems incredible in a station, the headquarters of a Cavalry Brigade. On further inquiry, it turned out that he was not authorized (on paper) to stock a single Thomas splint."

Again, "With reference to the subject of fractures, I must report that I am still being faced with the problem of the treatment of fractures with incomplete union in bad position, in which every available means has failed to keep fragments in an extended and reduced position. In most of them the utmost ingenuity bas been exhibited by medical officers in their efforts to splint and extend the fragnents. The patients arrive here with the skin of the limb exhibiting blisters or pressure sores, due to the application of successive forms of retentive apparatus or strapping. The underlying cause of failure in these cases is the fact that in a hot climate, not only will various strappings and glues fail to hold, but the general and local vitality being low, from sweat-gland infection, etc., the skin blisters 
and sloughs easily, so that each successive form or means of extension applied is only effective long enough to do harm, by permitting periodic movements at the fracture site. The remedy seems to be: $(a)$ The immediate transfer of all such cases to a hospital where they can be dealt with by open operation (? save the marik!); or $(b)$ the use of extension apparatus attached direct to bone."

This state of affairs impressed me very unfavourably, as I was fresh from experiences of war surgery, where equipment was available almost ad lib. Simple fractures were still being "plated" in those days. In some cases I got good results by the use of meat skewers, and knitting needles transfixing the bone fragments, and in one instance the local Ordnance Officer converted a metal saddle stirrup, into a very good calliper.

Some of us require to remember that a "particular fracture does not demand treatment on a particular splint (sealed pattern-Mark 4)," but that each fracture requires treatment on its particular merits. Sealed pattern splints still have their uses for first aid; there is no better splint in this class than the Thomas splint for use in the field. It is also the splint of choice for treatment of many leg fractures in the wards at a later stage. But, can we not say that we have now passed the stage of named and sealed pattern splints, and that the era of adaptable, mouldable, light and ambulatory splints, with transfixion wires and pins has come to stay? Bowlby stated that the mortality in the Great War from fractures of the femur alone (exclusive of those who died at home) was 40 per cent. It behoves us to consider seriously whether we cannot utilize these newer methods in the surgery of war. Provided that fracture cases were segregated in the earlier stages, I think we undoubtedly could. Our ranks will be swelled on mobilization with a generation of younger surgeons well trained in the later methods. Team work would be essential. I am not convinced that the objections usually put forward to the use of plaster of Paris in war surgery now apply. The objections are as follows :-

(1) Efficient extension is difficult to attain. With extension direct to bone, or transfixing pins incorporated in the plaster, this should not be difficult.

(2) Plaster becomes soiled by discharges. Agreed, but not a real objection. Can be prevented to a large extent by suitably protected windows, vaseline packing and celophane paint. What about Winnett Orr's method in the treatment of osteomyelitis. Granted it is malodorous!

(3) Efficient application is difficult, time-consuming and requires long experience. I do not agree on the whole. Read the books, or Colonel Weddell's excellent article in the Journal of October, 1936. Any surgeon who has not by now mastered plaster technique will get nowhere.

No-I think the real difficulty will be with compound fractures, and how soon, and under what conditions, surgeons will be able to tackle them. Indeed-one is often tempted to wonder under what conditions all of us 
will be working in the next war, now that the world's air arm has developed to such an extent.

I like to be an optimist, and somewhat of a visionary, and imagine that I see a Special Fracture Centre near the fighting line, with a number of teams working smoothly and methodically, and the cases being evacuated, each one with his damaged limb, confortably immobilized in plaster, labelled for the base or home and " not to be touched or dressed during the journey" (unless swelling necessitates bi-valving the splint).

We can learn much from the inspiration of men like Bohler and Winnett Orr, and can improve our methods as we go. There is economy and comfort in the principles of immobilization with early active movement, and in the knowledge that a patient's own serum is a good and inexpensive dressing. 